\title{
Association Between Difficulty with VA Patient-Centered Medical Home Model Components and Provider Emotional Exhaustion and Intent to Remain in Practice
}

\author{
Eric A. Apaydin, $P h D^{1,2}$, Danielle Rose, $P h D^{7}$, Lisa S. Meredith, $P h D^{1,2}$, \\ Michael McClean, BA' , Timothy Dresselhaus, $M D^{3,4}$, and Susan Stockdale, $P h D^{1,5}$
}

'Center for the Study of Healthcare Innovation, Implementation and Policy, VA Greater Los Angeles Healthcare System, 11301 Wilshire Blvd. (151), Los Angeles, CA, USA; ${ }^{2}$ RAND Corporation, Santa Monica, CA, USA; ${ }^{2}$ VA San Diego Healthcare System, San Diego, CA, USA; ${ }^{4}$ School of Medicine, University of California, San Diego, San Diego, CA, USA; 5 Department of Psychiatry and Biobehavioral Sciences, University of California Los Angeles, Los Angeles, CA, USA.

BACKGROUND: The patient-centered medical home $(\mathrm{PCMH})$ model is intended to improve primary care, but evidence of its effects on provider well-being is mixed. Investigating the relationships between specific $\mathrm{PCMH}$ components and provider burnout and potential attrition may help improve the efficacy of the care model.

OBJECTIVE: We analyzed provider attitudes toward specific components of PCMH in the Veterans Health Administration (VA) and their relation to emotional exhaustion (EE) - a central component of burnout-and intent to remain in VA primary care.

DESIGN: Logistic regression analysis of a cross-sectional survey.

SUBJECTS: 116 providers (physicians; nurse practitioners; physician assistants) in 21 practices between September 2015 and January 2016 in one VA region.

MAIN MEASURES: Outcomes: burnout as measured with the emotional exhaustion (EE) subscale of the Maslach Burnout Inventory and intent to remain in VA primary care for the next 2 years; predictors: difficulties with components of PCMH, demographic characteristics. KEY RESULTS: Forty percent of providers reported high $\mathrm{EE} \mathrm{(} \geq 27$ points) and $63 \%$ reported an intent to remain in VA primary care for the next 2 years. Providers reporting high difficultly with PCMH elements were more likely to report high $\mathrm{EE}$, for example, coordinating with specialists (odds ratio [OR] 8.32, 95\% confidence interval [CI] 3.5819.33), responding to EHR alerts (OR 6.88; 95\% CI 1.9324.43), and managing unscheduled visits (OR 7.53, 95\% CI 2.01-28.23). Providers who reported high EE were also $87 \%$ less likely to intend to remain in VA primary care.

CONCLUSIONS: To reduce EE and turnover in PCMH, primary care providers may need additional support and training to address challenges with specific aspects of the model.

Prior Presentations This research was presented at the 2019 Academy Health Annual Research Meeting and at the 2019 VA HSR\&D/QUERI National Conference.

Electronic supplementary material The online version of this article (https://doi.org/10.1007/s11606-020-05780-8) contains supplementary material, which is available to authorized users.

Received October 3, 2019

Accepted March 6, 2020

Published online April 6, 2020
KEY WORDS: burnout; health professions work force; patient-centered medical home; organizational change; surveys.

J Gen Intern Med 35(7):2069-75

DOI: $10.1007 / \mathrm{s} 11606-020-05780-8$

(c) Society of General Internal Medicine (This is a U.S. government work and not under copyright protection in the U.S.; foreign copyright protection may apply) 2020

\section{INTRODUCTION}

Burnout is a widespread psychological syndrome among healthcare workers in the USA. Twenty-three to $60 \%$ of physicians are burned out from chronic job-related stressors. ${ }^{1}$ While individual workers experience burnout through emotional exhaustion (EE), depersonalization, and low personal accomplishment, ${ }^{2}$ healthcare organizations experience its effects through lower productivity, increased turnover, lower quality of care, and worse relationships with patients. ${ }^{3-5}$ In addition, this decline in productivity and increase in turnover could exacerbate already existing shortages in the primary care workforce. ${ }^{6,7}$

The patient-centered medical home (PCMH) model was developed to improve the delivery of primary care ${ }^{8}$ and it has been suggested that the medical home can improve workforce well-being. ${ }^{9}$ However, evidence for the effectiveness of the model on reducing provider burnout is mixed. Several longitudinal analyses of PCMH implementation show that the model may actually increase provider burnout after 1 to 4 years. ${ }^{10-12}$ In contrast, a quasi-experimental analysis reported a 24-month reduction in the EE component of provider burnout relative to control clinics without PCMH, suggesting that context is key to the success of the model. ${ }^{13}$ An Agency for Healthcare Research and Quality-funded meta-analysis ${ }^{14}$ reported moderate strength of evidence that the PCMH care model improves patient satisfaction and increases preventative care, as well as low strength of evidence that the model improves staff satisfaction and reduces emergency department admissions.

The PCMH model in the Veterans Health Administration (VA) was implemented as Patient Aligned Care Teams (PACT) in 2010. PACT was intended to increase patient- 
centeredness by improving care elements such as patient continuity, access, care management, and care coordination through a variety of structural and process changes, including team-based care, patient health portals and telephone care, same-day appointments, nurse care management, and coordination between specialty and non-specialty care. ${ }^{15}$ Some research suggests that PCMH transformation in the VA was associated with more emotional exhaustion in the short term $(1-2 \text { years })^{16}$ than in the long term (5-6 years $)^{17}$ and that some structural components (such as participatory team decisionmaking, team task sharing, and adequate staffing) may be associated with lower burnout. ${ }^{18-20}$ Other work, however, has described a reduction in the EE component of burnout after more effective implementation of PCMH in the VA. ${ }^{21}$ Previous qualitative work has also identified short staffing after VA PCMH implementation as a contributor to provider burnout. ${ }^{22}$ Implementation of PCMH in the VA has also been previously linked to turnover, ${ }^{23}$ but the impact of individual PCMH components was not assessed.

This study highlights provider difficulties with a range of PCMH components and examines the association between those difficulties and provider EE and intent to remain in VA primary care for the following 2 years. Our study is the first quantitative examination of the cross-sectional association between difficulties with particular PCMH components and the EE component of burnout within the PCMH setting. Specifically, we analyze provider difficulty with components of the PCMH model. Of note, only one other study, ${ }^{19}$ focusing on provider the reliance on their team to complete tasks, assessed the relationships between specific elements of PCMH and the emotional exhaustion component of burnout as measured by the Maslach Burnout Inventory (MBI). All other analyses measured EE using a non-MBI scale, ${ }^{10-12,18,20}$ or measured EE using the MBI but only assessed the overall relationship between PCMH and EE. ${ }^{13}, 16,17,21$ Understanding whether and how specific difficulties with PCMH components may impact EE could facilitate development of interventions or strategies to address these challenges and improve provider EE.

\section{METHODS}

\section{Design and Participants}

We surveyed a cross-section of all PCMH primary care providers in all 23 VA-owned and operated primary care practices (serving over 5000 patients) in one VA administrative region between 2015 and 2016. This survey was part of a larger evaluation assessing VA PCMH implementation and has been described in more detail elsewhere. ${ }^{17}$

\section{Measures}

We assessed provider outcomes of the emotional exhaustion (EE) component of burnout and intent to remain in practice. EE was assessed by measuring the frequency of burnout- related feelings and attitudes using the nine-item EE subscale of the Maslach Burnout Inventory (never; a few times a year; every month; a few times a month; every week; a few times a week; every day). Scores from those nine items were summed, and three levels of $\mathrm{EE}^{24}$ were created for analysis: none/low (0-18 points); medium (19-26 points); high (27-54 points). For multivariate analyses, we created a binary variable indicating high EE vs. medium/low/none. Intent to remain in VA primary care practice for the next 2 years was assessed using a five-level ordered response scale (strongly disagree; disagree; neither disagree nor agree; agree; strongly agree). This item was dichotomized into strongly agree and agree vs. neither disagree nor agree, disagree, or strongly disagree.

Predictors of EE and intent to remain included 13 selfreported measures of challenges with PCMH model components. Respondents reported their difficulty level (not applicable; not at all difficult; somewhat difficult; very difficult) with the following: achieving continuity of care for patient panels; managing unscheduled visits/same-day patients; achieving full team staffing (i.e., 3 full-time equivalent [FTE] staff [RN, LPN/LVN, clerk] to 1 FTE provider [MD, NP, PA] per team); working in teams that can distribute tasks once staffed; achieving adequate panel support from health coaches; achieving adequate panel support from social workers; achieving adequate panel support from pharmacists; working with an administrative call center to receive patient phone messages; completing post-discharge follow-up (in-person or by phone calls); reviewing and responding to alerts, additional signer requests and reminders in the electronic health record; reviewing and answering patient email (secure messages); achieving access, coordinating, and sharing responsibility with specialists and subspecialists; identifying and implementing improvements to day-to-day panel care. These items were developed based on qualitative interviews with primary leaders and frontline providers and staff, as well as documentation and implementation guidance for the VA PCMH model. Additionally, items were pretested for face validity with a group of VA primary care providers and staff, and dichotomized into somewhat or very difficult (SVD) vs. not at all difficult (NAD) for analysis. Control variables included provider type (MD; NP; PA), gender, age, race/ethnicity, VA tenure, and clinic-level exposure to an evidence-based quality improvement intervention (EBQI) that was found to reduce the EE component of burnout. ${ }^{17}$ Missing and not applicable values in PCMH model component and control variables were imputed using information from all model variables, by multiple imputation with chained equations. Missing observations for outcome variables (EE and intent to remain) were not imputed.

\section{ANALYSES}

We examined distributions of each variable before beginning analyses. Multivariate logistic regression models estimated the 
relationship between binary versions of $\mathrm{EE}$ or intent to remain in practice and difficulty with each PCMH model component. Models controlled for covariates, included sampling weights, and adjusted for clustering by facility. Additional analyses of the relationships between control variables and outcomes (Table $\mathrm{S} 1$ ) and response rates by provider type and site (Table S2) are presented in the Supplementary Material. Complete results (including non-significant predictors) from models without imputation (Tables S3 and S4) and from our full models (Table S5) are also presented in this section.

\section{RESULTS}

One hundred and sixteen primary care providers (70 physicians; 30 nurse practitioners; 3 physicians assistants; 310 respondents invited; $37.4 \%$ response rate) in 21 (of 23) practices responded to our survey. Physicians were significantly less likely $(p<0.0001)$ to respond $(30 \%)$ as compared with nurse practitioners (55\%) and physician assistants (75\%), but response rate did not differ by PC clinic. Survey weights were created and used in our models to account for this difference. Over $40 \%$ of the sample reported high EE, and nearly twothirds reported an intent to remain in VA primary care for the next 2 years. Sample characteristics and frequencies of provider-reported difficulty with PCMH model components are reported in Tables 1 and 2, respectively.

In our multivariate models, the odds of EE were significantly higher for respondents reporting medium or high difficulty with the following components (Table 3; Figs. 1 and 2): managing unscheduled/same-day patients (odds ratio [OR] 7.53, 95\% confidence interval [CI] 2.01-28.23; $p=0.005$; predicted probability $[\mathrm{PP}] 66.71 \%$ SVD vs. $30.76 \%$ NAD); achieving adequate panel support from pharmacists (OR 9.52, 95\% CI $2.66-34.03 ; p=0.002$; PP 78.33\% SVD vs. $35.68 \%$ NAD); working with an administrative call center (OR 3.20, 95\% CI $1.08-9.50 ; p=0.04$; PP $54.54 \%$ SVD vs. $31.71 \%$ NAD); reviewing and responding to electronic health record alerts (OR 6.88; 95\% CI 1.93-24.43; $p=0.005$; PP 65.26\% SVD vs. $29.43 \% \mathrm{NAD}$ ); reviewing and answering patient email (OR 10.81; 95\% CI 3.47-33.65; $p<0.0001$; PP $78.22 \%$ SVD vs. $35.72 \%$ NAD); achieving access, coordinating, and sharing responsibility with specialists (OR 8.32, 95\% CI $3.58-19.33 ; p<0.0001$; PP $65.74 \%$ SVD vs. $28.49 \%$ NAD); and identifying and implementing improvements to day-to-day panel care (OR 6.93; 95\% CI 1.93-24.89; $p=$ 0.006; PP $72.80 \%$ SVD vs. $36.32 \%$ NAD).] $\rightarrow$ ] $\rightarrow$

Respondents were also less likely to report an intent to remain in VA practice if they reported medium or high difficulty with the following: managing unscheduled/same-day patients (OR 0.18 ; 95\% CI $0.05-0.65 ; p=0.01$; PP $38.11 \%$ SVD vs. $73.96 \%$ NAD); achieving full team staffing (OR $0.29,95 \%$ CI $0.09-0.92 ; p=0.04$; PP $46.50 \%$ SVD vs. $72.82 \% \mathrm{NAD}$; and reviewing and answering patient email
Table 1 Characteristics of the Sample $(n=116)$

\begin{tabular}{lc}
\hline \hline Characteristic & $\boldsymbol{n}(\boldsymbol{\%})$ \\
\hline Provider type & \\
Physician (MD or DO) & $70(68)$ \\
Nurse practitioner & $30(29)$ \\
Physician assistant & $3(3)$ \\
Gender & \\
Male & $42(42)$ \\
Female & $57(58)$ \\
Age & \\
30-39 years old & $13(14)$ \\
40-49 years old & $30(31)$ \\
50-59 years old & $27(28)$ \\
60+ years old & $26(27)$ \\
Race & \\
White & $61(53)$ \\
Non-white & $55(47)$ \\
Years in VA practice & \\
<5 years & $49(45)$ \\
5-9 years & $14(13)$ \\
10-14 years & $17(15)$ \\
15+ years & $30(27)$ \\
Received evidence-based quality improvement intervention (EBQI) \\
Yes & $56(48)$ \\
No & $60(52)$ \\
$\quad$ Emotional exhaustion component of burnout (Maslach Burnout \\
None/low (0-18) & \\
Medium (19-26) & $50(45)$ \\
High (27-54) & $16(14)$ \\
Intent to remain in VA primary care for the next 2 years & $46(41)$ \\
Strongly disagree & $7(6)$ \\
Disagree & $7(6)$ \\
Neither disagree nor agree & $28(25)$ \\
Agree & $47(42)$ \\
Strongly agree & $24(21)$ \\
\hline
\end{tabular}

Note: Values are missing for some characteristics; column percentages were calculated with denominators of only non-missing values for each characteristic

(OR $0.16,95 \%$ CI $0.04-0.67 ; p=0.02 ;$ PP $28.73 \%$ SVD vs. $68.59 \%$ NAD).

Finally, providers reporting high EE were $87 \%$ less likely to report an intention to remain in VA primary care practice for the next 2 years (OR $0.13,95 \%$ CI $0.05-0.34 ; p<0.0001$; PP $37.64 \%$ SVD vs. $79.91 \%$ NAD).

\section{DISCUSSION}

This study found that over $40 \%$ of VA primary care providers participating in the patient-centered medical home model in one regional healthcare network reported high levels of EE, and over a third did not intend to remain in VA practice for the next 2 years. In our cross-sectional analysis, primary care providers were more likely to report $\mathrm{EE}$ or a lower intent to remain in VA practice if they found medium or high difficulty with managing unscheduled patients, achieving full team staffing, achieving adequate panel support from pharmacists, working with an administrative call center, responding to EHR alerts, answering patient email, coordinating with specialists, and identifying and implementing improvements for day-today panel care.

Our findings show that administrative work, excessive overall workload, inadequate care coordination, and poor 
Table 2 Provider-Reported Difficulties with PCMH Model Components

\begin{tabular}{|c|c|c|c|c|c|}
\hline \multirow[t]{2}{*}{$\overline{\text { PCMH model component }}$} & \multirow[b]{2}{*}{$\begin{array}{l}\text { Total responses } \\
\text { (n) }\end{array}$} & \multicolumn{4}{|c|}{ Provider-reported difficulty ( $n$ [row \%]) } \\
\hline & & $\begin{array}{l}\text { Not } \\
\text { applicable }\end{array}$ & $\begin{array}{l}\text { Not at all } \\
\text { difficult }\end{array}$ & $\begin{array}{l}\text { Somewhat } \\
\text { difficult }\end{array}$ & $\begin{array}{l}\text { Very } \\
\text { difficult }\end{array}$ \\
\hline Achieving continuity of care for patient panels & 110 & $2(2)$ & $67(61)$ & $33(30)$ & $8(7)$ \\
\hline Managing unscheduled visits/same-day patients & 111 & $1(1)$ & $29(26)$ & $49(44)$ & $32(29)$ \\
\hline Achieving full team staffing & 111 & $4(4)$ & $25(23)$ & $42(38)$ & $40(36)$ \\
\hline Working in teams once staffed & 111 & $6(5)$ & $61(55)$ & $33(30)$ & $11(10)$ \\
\hline Achieving adequate panel support from health coaches & 110 & $31(28)$ & $30(27)$ & $25(23)$ & $24(22)$ \\
\hline Achieving adequate panel support from social workers & 111 & $3(3)$ & $66(59)$ & $27(24)$ & $15(14)$ \\
\hline Achieving adequate panel support from pharmacists & 111 & $3(3)$ & $72(65)$ & $23(21)$ & $13(12)$ \\
\hline Working with an administrative call center & 111 & $11(10)$ & $17(15)$ & $43(39)$ & $40(36)$ \\
\hline Completing post-discharge follow-up & 111 & $8(7)$ & $54(49)$ & $43(39)$ & $6(5)$ \\
\hline $\begin{array}{l}\text { Reviewing and responding to electronic health record } \\
\text { alerts }\end{array}$ & 111 & $1(1)$ & $37(33)$ & $41(37)$ & $32(29)$ \\
\hline Reviewing and answering patient email & 111 & $1(1)$ & $61(55)$ & $35(32)$ & $14(13)$ \\
\hline $\begin{array}{l}\text { Achieving access, coordinating, and sharing } \\
\text { responsibility with specialists }\end{array}$ & 111 & $2(2)$ & $20(18)$ & $57(51)$ & $32(29)$ \\
\hline $\begin{array}{l}\text { Identifying and implementing improvements to day-to- } \\
\text { day panel care }\end{array}$ & 111 & $2(2)$ & $45(41)$ & $51(46)$ & $13(12)$ \\
\hline
\end{tabular}

Note: Frequencies of only non-missing values are presented here. PCMH, patient-centered medical home

staffing are associated with emotional exhaustion. These results are largely consistent with what has been reported in the literature. Providers in our sample who reported having difficulty with the administrative tasks of responding to patient emails or phone messages (via the administrative call center) were more likely to report EE. Administrative work ${ }^{25,26}$ and electronic health records, especially with poor usability, ${ }^{27,} 28$ have been consistently linked with lower provider satisfaction and higher burnout. ${ }^{29,}{ }^{30}$ Workload-related difficulties in managing unscheduled patients and day-to-day panel care were also both linked to higher EE in our sample. In several analyses of PCMH in other settings, excessive workload been consistently linked to higher provider burnout ${ }^{31-33}$ and is partially linked to burnout in one analysis of VA's PCMH. ${ }^{34}$ Our results show that difficulties in care coordination between VA PCMH providers and specialists (both through EHR alerts

Table 3 Odds and Predicted Probabilities of Emotional Exhaustion and Intent to Remain by Difficulties with PCMH Model Components

\begin{tabular}{|c|c|c|}
\hline \multirow[t]{2}{*}{ PCMH model component } & \multirow{2}{*}{$\frac{\mathrm{EE}(n=110)}{\mathrm{OR}(95 \% \mathrm{CI})}$} & \multirow{2}{*}{$\begin{array}{l}\text { Intent to remain }(n=111) \\
\text { OR }(95 \% \mathrm{CI})\end{array}$} \\
\hline & & \\
\hline \multicolumn{3}{|c|}{ Managing unscheduled visits/same-day patients } \\
\hline Not at all difficult & Ref. & Ref. \\
\hline Somewhat or very difficult & $7.53(2.01-28.23)^{*}$ & $0.18(0.05-0.65)^{*}$ \\
\hline \multicolumn{3}{|l|}{ Achieving full team staffing } \\
\hline Not at all difficult & Ref. & Ref. \\
\hline Somewhat or very difficult & $3.12(0.86-11.31)$ & $0.29(0.09-0.92)^{*}$ \\
\hline \multicolumn{3}{|c|}{ Achieving adequate panel support from pharmacists } \\
\hline Not at all difficult & Ref. & Ref. \\
\hline Somewhat or very difficult & $9.52(2.66-34.03) *$ & $0.34(0.07-1.66)$ \\
\hline \multicolumn{3}{|c|}{ Working with an administrative call center } \\
\hline Not at all difficult & Ref. & Ref. \\
\hline Somewhat or very difficult & $3.20(1.08-9.50)^{*}$ & $0.64(0.20-2.03)$ \\
\hline \multicolumn{3}{|c|}{ Reviewing and responding to electronic health record alerts } \\
\hline Not at all difficult & Ref. & Ref. \\
\hline Somewhat or very difficult & $6.88(1.93-24.43) *$ & $0.36(0.12-1.07)$ \\
\hline \multicolumn{3}{|c|}{ Reviewing and answering patient email } \\
\hline Not at all difficult & Ref. & Ref. \\
\hline Somewhat or very difficult & $10.81(3.47-33.65)^{*}$ & $0.16(0.04-0.67)^{*}$ \\
\hline \multicolumn{3}{|c|}{ Achieving access, coordinating, and sharing responsibility with specialists and subspecialists } \\
\hline Not at all difficult & Ref. & Ref. \\
\hline Somewhat or very difficult & $8.32(3.58-19.33)^{*}$ & $0.52(0.21-1.29)$ \\
\hline \multicolumn{3}{|c|}{ Identifying and implementing improvements to day-to-day panel care } \\
\hline Not at all difficult & Ref. & Ref. \\
\hline Somewhat or very difficult & $6.93(1.93-24.89) *$ & $0.26(0.06-1.14)$ \\
\hline
\end{tabular}

Note: Models controlled for provider type, provider gender, provider age, provider race/ethnicity, provider tenure, and facility receipt of EBQI, included sampling weights and were clustered by facility. Missing values for component difficulty, provider type, provider gender, provider age, provider race/ethnicity, provider tenure, and facility receipt of EBQI variables were imputed by multiple imputation by chained equations. CI, confidence interval; EBQI, evidence-based quality improvement intervention; EE, emotional exhaustion component of burnout; OR, odds ratio; $P C M H$, patient-centered medical home

$*_{p}<0.05$ for very difficult or somewhat difficult $v$ s. not at all difficult 


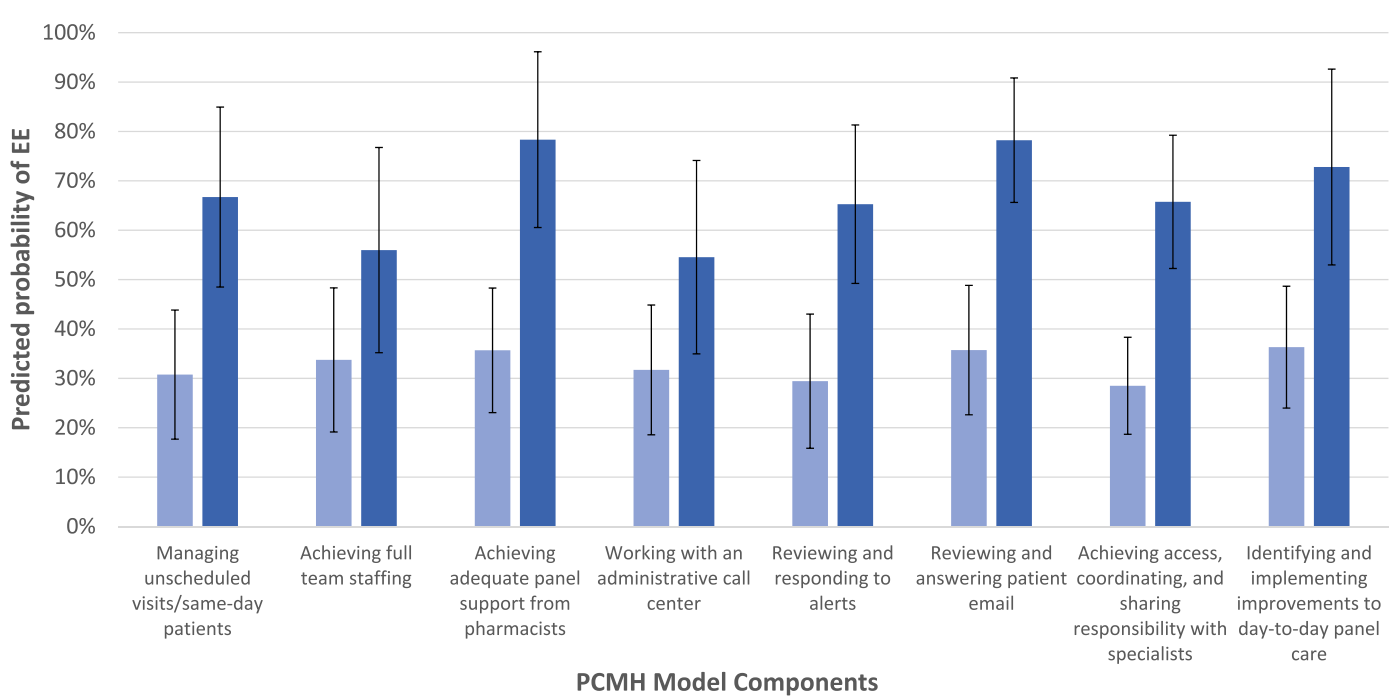

Difficulty with PCMH model component $\quad$ Not at all difficult $\quad$ Somewhat or very difficult

Figure 1 Predicted probabilities of emotional exhaustion by difficulties with patient-centered medical home model components. Note: Error bars represent $95 \%$ confidence intervals for predicted probabilities. EE, emotional exhaustion component of burnout; PCMH, patient-centered medical home.

and traditional means of communication) were associated with higher EE. In the medical home model, better care coordination has been associated with lower provider EE. ${ }^{13,21}$ Finally, we show that difficulties in achieving adequate panel support from pharmacists was also linked to higher EE. Shortages in staffing have also been consistently linked to burnout in the VA PCMH model ${ }^{18,34}$ and in another setting. ${ }^{31}$

This work expands on previous studies of the impact of the PCMH model on workforce experience in that we are the first to present the relationship between a range of PCMH components and intent to remain in practice. In particular, we show relationships between difficulties with managing unscheduled patients, achieving full team staffing, and answering patient email with lower intent to remain. The association between burnout and lower intent to remain or reduction in clinical hours has been highlighted in previous non-PCMH settings, $5,35,36$ but our work is the first to emphasize the relationship between difficulties with specific PCMH model components and intent to remain. Further descriptive and interventional work is needed on the relationship between $\mathrm{EE}$ and intent to remain, with an eye toward potential remedies (e.g., reductions in clinical hours) to mitigate turnover when possible.

This analysis had several limitations: (1) the sample size is relatively small; (2) data were imputed, which can introduce bias into models, but sensitivity analyses showed that after imputation, only one additional PCMH model component was related to each outcome, and one was no longer related to EE; (3) the data are cross-

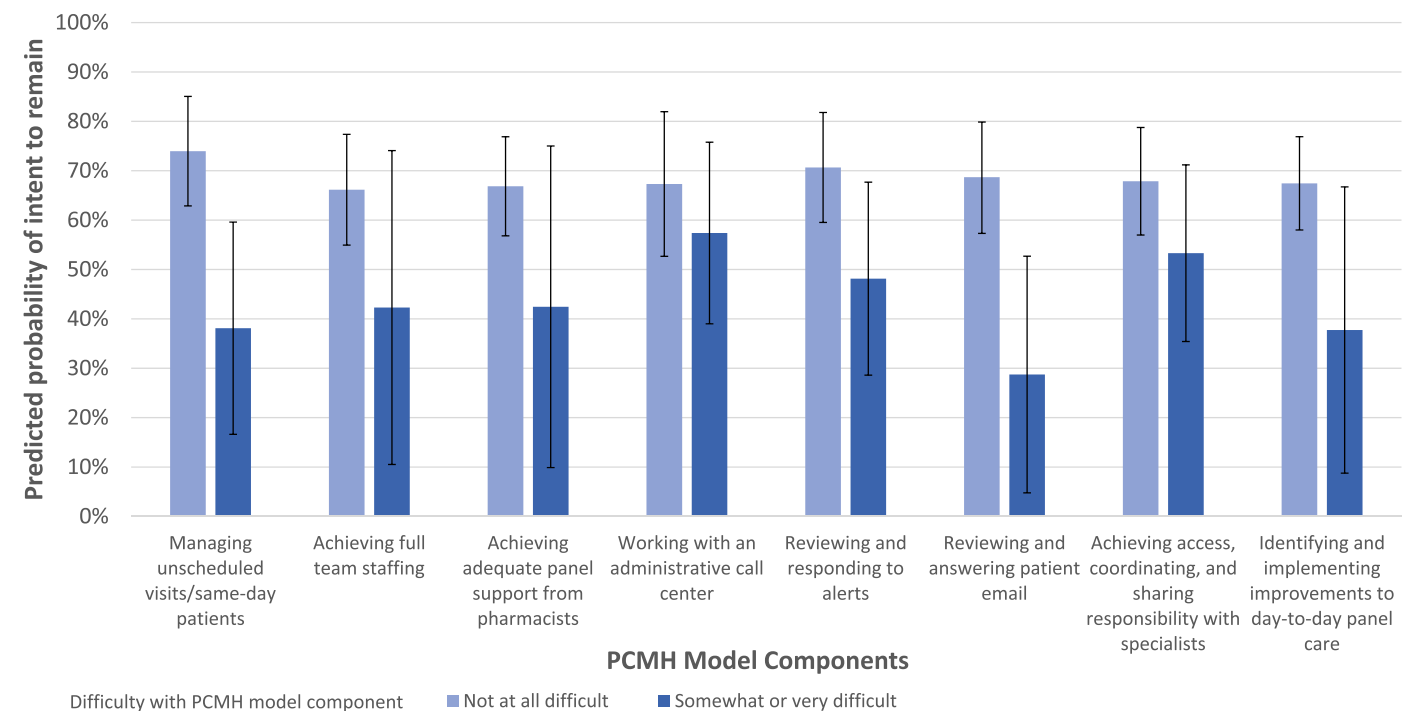

Figure 2 Predicted probabilities of intent to remain by difficulties with patient-centered medical home model components. Note: Error bars represent $95 \%$ confidence intervals for predicted probabilities. PCMH, patient-centered medical home. 
sectional, and thus, we could not determine the causal direction of the association; (4) self-reported difficulty with PCMH model components may represent a general difficulty with the model overall, but our results suggest a starting point for future qualitative studies to explore these difficulties in more depth; (5) several PCMH model component items are "double-barreled," which may have affected validity of these items; (6) this study may not be generalizable outside of VA, in other VA regions, or outside of other large, integrated healthcare systems.

These limitations are balanced by several strengths of this work. Despite a small sample, we identified a number of PCMH model components associated with $\mathrm{EE}$ and intent to remain. We also measured burnout using the comprehensive emotional exhaustion subscale of the MBI, while many previous studies ${ }^{10-12 \text {, }}$ 18,20 used a single-item measure of the outcome. Finally, we present the first analysis linking EE and PCMH model components to intent to remain in a PCMH setting.

Strategies aimed at both the individual and organizational level may be needed to prevent and/or alleviate burnout. ${ }^{17-19,29,34}$ The VA PCMH model was centrally developed and implemented in a consistent manner, without specific provisions for provider input. ${ }^{15}$ Provider difficulties with specific components of the VA PCMH model may have been avoided if the model were developed with provider input. Interventions facilitating provider-involved quality improvement have consistently decreased burnout, both inside VA PCMH teams ${ }^{17,37}$ and in other settings. ${ }^{38,}$ ${ }^{39}$ Healthcare systems with team-based care models such as PCMH should routinely measure and track provider burnout and solicit feedback from providers for improving PCMH model execution. Previous authors have commented $^{40}$ and shown ${ }^{21,41,42}$ that PCMH model fidelity, and not just the intervention alone, is crucial to the model's success. Incorporating provider input in quality improvement initiatives for PCMH may prevent EE, and in turn, potentially reduce provider turnover and associated costs.

\section{Contributors: None}

Corresponding Author: Eric A. Apaydin, PhD; Center for the Study of Healthcare Innovation, Implementation and Policy VA Greater Los Angeles Healthcare System, 11301 Wilshire Blvd. (151), Los Angeles, CA 90073, USA (e-mail: eric.apaydin@va.gov).

Funding Information This analysis was funded by the Department of Veterans Affairs (VA) Office of Primary Care (\#XVA 65-018). Dr. Apaydin was additionally supported by the VA Office of Academic Affiliations through the Advanced Fellowship in Health Services Research \& Development.

\section{Compliance with Ethical Standards:}

Conflict of Interest: The authors declare that they do not have a conflict of interest.
Disclaimer: The contents of this article do not represent the views of the US Department of Veterans Affairs or the United States Government.

\section{REFERENCES}

1. Rotenstein LS, Torre M, Ramos MA, et al. Prevalence of burnout among physicians: a systematic review. JAMA. 2018;320(11):1131-1150.

2. Maslach C, Leiter MP. Understanding the burnout experience: recent research and its implications for psychiatry. World Psychiatry. 2016;15(2): 103-111.

3. Noseworthy J, Madara J, Cosgrove D, et al. Physician burnout is a public health crisis: a message to our fellow health care CEOs. Health Affairs Blog. 2017. Available at: https://www.healthaffairs.org/do/10.1377/ hblog20170328.059397/full/. Accessed 18 Mar 2020.

4. Panagioti M, Geraghty K, Johnson J, et al. Association between physician burnout and patient safety, professionalism, and patient satisfaction: a systematic review and meta-analysis. JAMA Intern Med. 2018;178(10):1317-1330.

5. Shanafelt TD, Mungo M, Schmitgen J, et al. Longitudinal study evaluating the association between physician burnout and changes in professional work effort. Mayo Clin Proc. 2016;91(4):422-431.

6. Spinelli WM. The phantom limb of the triple aim. Mayo Clin Proc. 2013;88(12):1356-1357.

7. Bodenheimer T, Sinsky C. From triple to quadruple aim: care of the patient requires care of the provider. Ann Fam Med. 2014;12(6):573-576.

8. American Academy of Family Physicians, American Academy of Pediatrics, American College of Physicians, American Osteopathic Association. Joint principles of the patient-centered medical home. 2007. Available at: https://www.aafp.org/dam/AAFP/documents/practice_management/pcmh/initiatives/PCMHJoint.pdf. Accessed 18 Mar 2020.

9. Bodenheimer T, Laing BY. The teamlet model of primary care. Ann Fam Med. 2007;5(5):457-461.

10. Lewis SE, Nocon RS, Tang $\mathrm{H}$, et al. Patient-centered medical home characteristics and staff morale in safety net clinics. Arch Intern Med. 2012; 172(1):23-31.

11. Nutting PA, Crabtree BF, Miller WL, Stange KC, Stewart E, Jaén C. Transforming physician practices to patient-centered medical homes: lessons from the national demonstration project. Health Aff (Millwood). 2011;30(3):439-445.

12. Nocon RS, Fairchild PC, Gao Y, et al. Provider and staff morale, job satisfaction, and burnout over a 4-year medical home intervention. J Gen Intern Med. 2019;34(6):952-959.

13. Reid RJ, Coleman K, Johnson EA, et al. The Group Health medical home at year two: cost savings, higher patient satisfaction, and less burnout for providers. Health Aff (Millwood). 2010;29(5):835-843.

14. Jackson GL, Powers BJ, Chatterjee R, et al. The patient-centered medical home: a systematic review. Ann Intern Med. 2013;158(3):169-178.

15. Rosland AM, Nelson K, Sun H, et al. The patient-centered medical home in the Veterans Health Administration. Am $J$ Manag Care. 2013;19(7):e263-272.

16. Meredith LS, Schmidt Hackbarth N, Darling J, et al. Emotional exhaustion in primary care during early implementation of the VA's medical home transformation: Patient-aligned Care Team (PACT). Med Care. 2015;53(3):253-260.

17. Meredith LS, Batorsky B, Cefalu M, et al. Long-term impact of evidencebased quality improvement for facilitating medical home implementation on primary care health professional morale. BMC Fam Pract. 2018; 19(149): 1-10.

18. Helfrich CD, Dolan ED, Simonetti J, et al. Elements of team-based care in a patient-centered medical home are associated with lower burnout among VA primary care employees. J Gen Intern Med. 2014;29(Suppl 2):S659-666.

19. Kim LY, Rose DE, Soban LM, et al. Primary care tasks associated with provider burnout: findings from a Veterans Health Administration survey. J Gen Intern Med. 2018;33(1):50-56.

20. Edwards ST, Helfrich CD, Grembowski D, et al. Task delegation and burnout trade-offs among primary care providers and nurses in Veterans Affairs Patient Aligned Care Teams (VA PACTs). J Am Board Fam Med. 2018;31(1):83-93.

21. Nelson KM, Helfrich $\mathrm{C}$, Sun H, et al. Implementation of the patientcentered medical home in the Veterans Health Administration: associations with patient satisfaction, quality of care, staff burnout, and hospital 
and emergency department use. JAMA Intern Med. 2014;174(8):13501358.

22. Chuang E, Brunner J, Mak S, et al. Challenges with implementing a patient-centered medical home model for women veterans. Womens Health Issues. 2017;27(2):214-220.

23. Sylling PW, Wong ES, Liu CF, et al. Patient-centered medical home implementation and primary care provider turnover. Med Care. 2014;52(12):1017-1022.

24. Maslach C, Leiter MP. Maslach Burnout Inventory Manual. Palo Alto, CA: Consulting Psychologists Press; 1996.

25. Landon BE, Aseltine R, Shaul JA, Miller Y, Auerbach BA, Cleary PD. Evolving dissatisfaction among primary care physicians. Am J Manag Care. 2002;8(10):890-901.

26. Ly DP, Glied SA. The impact of managed care contracting on physicians. J Gen Intern Med. 2014;29(1):237-242.

27. Babbott S, Manwell LB, Brown R, et al. Electronic medical records and physician stress in primary care: results from the MEMO Study. J Am Med Inform Assoc. 2014;21(e1):e100-106.

28. Friedberg MW, Chen PG, Van Busum KR, et al. Factors affecting physician professional satisfaction and their implications for patient care, health systems, and health policy. Santa Monica, CA: RAND Corporation;2013.

29. Shanafelt TD, Dyrbye LN, Sinsky C, et al. Relationship between clerical burden and characteristics of the electronic environment with physician burnout and professional satisfaction. Mayo Clin Proc. 2016;91(7):836848

30. Love JS, Wright A, Simon SR, et al. Are physicians' perceptions of healthcare quality and practice satisfaction affected by errors associated with electronic health record use? J Am Med Inform Assoc. 2012;19(4):610-614.

31. Deckard G, Meterko M, Field D. Physician burnout: an examination of personal, professional, and organizational relationships. Med Care. 1994;32(7):745-754.

32. Gregory ST, Menser T. Burnout among primary care physicians: a test of the Areas of Worklife Model. J Health Manag. 2015;60(2):133-148.
33. Linzer M, Manwell LB, Williams ES, et al. Working conditions in primary care: physician reactions and care quality. Annals of Internal Medicine. 2009;151(1):28-36.

34. Helfrich CD, Simonetti JA, Clinton WL, et al. The association of teamspecific workload and staffing with odds of burnout among VA primary care team members. J Gen Intern Med. 2017;32(7):760-766.

35. Hoff T, Whitcomb WF, Nelson JR. Thriving and surviving in a new medical career: the case of hospitalist physicians. J Health Soc Behav. 2002;43(1):72-91.

36. Shanafelt TD, West CP, Sloan JA, et al. Career fit and burnout among academic faculty. Arch Intern Med. 2009;169(10):990-995.

37. Warde C, Ching WT, Sohmer R. Enhancing resilience to promote VA patient-centered medical home transformation and the untoward effects of the national access crisis. J Gen Intern Med. 2015;30(Suppl 2):516.

38. Linzer M, Poplau S, Grossman E, et al. A cluster randomized trial of interventions to improve work conditions and clinician burnout in primary care: results from the Healthy Work Place (HWP) Study. J Gen Intern Med. 2015;30(8):1105-1111.

39. Dunn PM, Arnetz BB, Christensen JF, Homer L. Meeting the imperative to improve physician well-being: assessment of an innovative program. J Gen Intern Med. 2007;22(11):1544-1552.

40. Jackson GL, Williams JW. Does PCMH "work"?-the need to use implementation science to make sense of conflicting results. JAMA Intern Med. 2015;175(8): 1369-1370.

41. Tuepker A, Kansagara D, Skaperdas E, et al. "We've not gotten even close to what we want to do": a qualitative study of early patient-centered medical home implementation. J Gen Intern Med. 2014;29(Suppl 2):614622

42. Solimeo SL, Ono SS, Lampman MA, Paez MB, Stewart GL. The empowerment paradox as a central challenge to patient centered medica home implementation in the veteran's health administration. J Interprof Care. 2015;29(1):26-33.

Publisher's Note: Springer Nature remains neutral with regard to jurisdictional claims in published maps and institutional affiliations. 